\title{
Bringing Emergency Neurology to Ambulances: Mobile Stroke Unit
}

\author{
Ritvij Bowry, MD ${ }^{1}$ James C. Grotta, MD² \\ 1 Department of Neurology and Neurosurgery, McGovern Medical \\ School, University of Texas Health Science Center at Houston, \\ Houston, Texas \\ 2 Mobile Stroke Unit and Stroke Research, Clinical Innovation and \\ Research Institute, Memorial Hermann-Texas Medical Center, \\ Houston, Texas
}

\begin{abstract}
Address for correspondence James C. Grotta, MD, Mobile Stroke Unit and Stroke Research, Clinical Innovation and Research Institute, Memorial Hermann-Texas Medical Center, 6410 Fannin St, Suite 1423, Houston, TX 77030 (e-mail: james.c.grotta@uth.tmc.edu).
\end{abstract}

Semin Respir Crit Care Med 2017;38:713-717.

\begin{abstract}
Keywords

- stroke

- thrombectomy

- telemedicine

- thrombolysis

- prehospital

- ambulance

- neurological emergencies

Ischemic stroke results from blocked arteries in the brain, with earlier thrombolysis with intravenous tissue plasminogen activator (tPA) and/or mechanical thrombectomy resulting in improved clinical outcomes. Mobile Stroke Unit (MSU) can speed up the treatment with tPA and facilitate faster triage for patients to hospitals for mechanical thrombectomy. The first registry-based MSU study in Germany demonstrated faster treatment times with tPA using a MSU, a higher proportion of patients being treated within the first "golden hour," and a suggestion of improved 3-month clinical outcomes. The first multicenter, prospective, randomized clinical trial comparing MSU versus standard care was started in 2014 after the launch of the MSU in Houston, TX, demonstrating the feasibility and safety of MSU operation in the United States, and reliability of telemedicine to evaluate stroke patients for tPA eligibility. Although conclusive evidence from clinical trials to support MSUs as being cost effective and improving clinical outcomes is still needed, there are a myriad of other clinical and research applications of MSUs that could have profound implications for managing patients with neurological emergencies.
\end{abstract}

\section{Background}

A blocked artery in the brain can often lead to irreversible cell death resulting in an ischemic stroke with major disability or death. ${ }^{1,2}$ Permanent damage to brain tissue (infarct core) occurs within minutes, with adjacent regions consisting of areas of brain where blood flow and oxygen are compromised (ischemic penumbra), but still salvageable. Timely reperfusion of this penumbra is the mainstay of a "time-isbrain" approach for treating such strokes, with the ultimate goal of treatment consisting of faster and complete restoration of blood flow of occluded arteries to save brain cells from irreparable injury.

The standard treatment for acute ischemic stroke involves the administration of intravenous tissue plasminogen acti- vator (IV tPA) within 4.5 hours of symptom onset, and/or with intra-arterial mechanical thrombectomy for selected patients with large vessel arterial occlusions. ${ }^{3}$ Not only is earlier recanalization associated with improved outcomes ${ }^{4-10}$ but there is a $15 \%$ incremental decrease in chances of a good clinical outcome for every 30-minute reperfusion delay with intra-arterial treatment. ${ }^{10}$

Despite the potential for IV tPA to improve outcomes for patients with ischemic strokes, however, only a limited number of patients are able to receive such treatment in a timely manner. ${ }^{11,12}$ While reasons for this discrepancy are complex, major obstacles include time delays in patients' arrival to hospitals capable of administering such treatment. ${ }^{13,14}$ In some estimates, the median door to needle
Issue Theme Advancements in Neurocritical Care and Emergency Neurology; Guest Editors: David Y. Hwang, MD, FNCS, and David M. Greer, MD, MA, FCCM, FAHA, FNCS, FAAN, FANA
Copyright $\odot 2017$ by Thieme Medical Publishers, Inc., 333 Seventh Avenue, New York, NY 10001, USA.

Tel: +1(212) 584-4662.
DOI https://doi.org/ 10.1055/s-0037-1607994. ISSN 1069-3424. 
time in stroke center emergency rooms in the United States is approximately 60 minutes, ${ }^{15}$ with most patients being treated beyond 2 hours. ${ }^{16}$ Given the direct relationship of improved outcomes with faster treatment times with IV $\mathrm{tPA}^{4-9}$ minimizing delays in the triage, evaluation, and treatment of patients is vital.

Hospital notification by emergency medical system (EMS) units prior to hospital arrival is key to faster triage and treatment of stroke patients, leading to more efficient mobilization of medical teams and initiation of hospital procedures. ${ }^{17}$ Administrative delays in the triage process of stroke patients in the emergency room often offset this potential time savings, however, irrespective of the time needed to conduct a history and physical, obtain a computed tomography (CT) scan, and initiate treatment with IV tPA.

Considering the association between faster tPA administration (within the first 60 minutes after symptom onset, the "golden hour") and a higher frequency of discharge home (rather than to a nursing facility or other institutions ${ }^{18}$ ) and better outcomes at discharge, ${ }^{19}$ optimization of stroke care is imperative. The use of specially equipped ambulances in the prehospital setting is one way to facilitate the earlier treatment of stroke patients. While studies have reported the feasibility of telestroke assessment in ambulances with regard to various time metrics related to thrombolysis and triage, ${ }^{20,21}$ evidence to support the impact on clinical outcomes and health care cost savings has been lacking.

\section{The Mobile Stroke Unit Concept}

Fassbender et al in Homburg, Germany, first proposed ${ }^{22}$ and performed $^{23}$ the Mobile Stroke Unit (MSU) concept (ambulance equipped with a CT scanner, laboratory testing, telemedicine [TM] connection to the hospital, and appropriate medication on-board [OB] the MSU) enabling the prehospital diagnosis and treatment of stroke in a relatively rural setting. Results from their study demonstrated that prehospital management achieved a median symptom onset-to-therapy decision time of 56 minutes (vs. 104 for hospital-based intervention, $p<0.0001$ ) and a median symptom onsetto-treatment time of 72 minutes (vs. 153, $p=0.0011$ ) without any concerns of safety resulting from the use of a MSU. More than $50 \%$ of patients had a time-to-therapy decision within the first "golden hour" from stroke symptom onset. ${ }^{24}$

Similar trends in safety and time savings (time from initial call to treatment) were seen in the Pre-Hospital Acute Neurological Treatment and Optimization of Medical care in Stroke (PHANTOM-S) study undertaken in an urban environment by the Stroke Emergency Mobile Unit (STEMO) group in Berlin, Germany ${ }^{25}$ with an increased likelihood for discharge home for patients who received thrombolysis within the first 60 minutes from stroke onset. ${ }^{18}$ An observational, registry-based comparison of 3-month functional outcomes using modified Rankin scale (mRS) scores suggested a beneficial effect of prehospital thrombolysis using the MSU, compared with standard hospital-based administration of tPA (odds ratio [OR]: $1.40,95 \%$ confidence interval [CI]: $1.00-1.97 ; p=0.052$ for $m R S \leq 1$ ), with a time saving of $\sim 30$ minutes to the initiation of thrombolysis. ${ }^{26}$ The STEMO group is currently conducting a prospective study employing a city-wide coverage scheme consisting of several MSUs working in conjunction with local EMS services of the fire department.

Following the successes of establishing and operating the first MSUs in Germany, the earlier treatment of ischemic strokes became a reality in the United States with the launch of the first MSU in Houston, TX, in May $2014 .{ }^{27}$ Following the initial "run-in" phase, ${ }^{28}$ the reliability of a TM-based vascular neurologist (VN) to determine tPA eligibility compared with an OB neurologist was established, ${ }^{29}$ along with demonstrable radiation safety of operating a portable CT scanner (radiation levels were also found to be within normal limits in the MSUs in Germany). ${ }^{30}$ A prospective, multicenter, cluster-randomized comparative effectiveness trial (Benefits of Stroke Treatment Delivered Using a MSU compared with standard management by emergency medical services) is currently ongoing, which is expected to answer relevant questions regarding clinical benefit and cost effectiveness, including poststroke health care utilization and fixed costs of introducing and operating an MSU. ${ }^{31}$ The initial establishment in Houston was followed by the implementation of similar MSU programs across various other sites including Cleveland, Denver, Memphis, Toledo, New York City, New Jersey, Pennsylvania, Phoenix, Chicago, Los Angeles, and Indianapolis, with plans to launch similar programs globally in cities such as Paris (France), Edmonton (Alberta, Canada), and Melbourne (Australia).

\section{The Nuts and Bolts of Setting Up a Mobile Stroke Unit}

The MSU itself is a standard ambulance that is fitted with a portable CT scanner, and consists of all necessary equipment needed for the acute evaluation and treatment of a stroke patient. The MSU team can be composed of either an OB- or TM-based VN, a nurse skilled in stroke management, a paramedic, and a CT technologist. While the first prehospital thrombolysis projects relied on physician-staffed ambulances, ${ }^{23,25,27,28}$ the group in Cleveland showed that a model using solely a TM VN on a MSU is feasible and associated with a low technical failure rate (all technical delays were $<1$ minute without affecting patient care). The replacement of an OB VN with a TM VN might not only lead to savings in personnel costs but could also facilitate the simultaneous operation of multiple MSUs by a single TM VN in geographically distinct areas. ${ }^{21}$ Subsequently, the Houston team demonstrated the reliability between an OB VN and a TM VN in making clinical decisions regarding to tPA eligibility, ${ }^{29}$ which was demonstrably comparable to two VNs seeing the same patient in an emergency department. $^{32}$ Time-to-tPA decision (OB: 18 minutes [interquartile range, IQR: 14-23] vs. TM: 21 minutes [IQR: 16-26], $p=0.05$ ) and initiation of tPA bolus were equivalent (OB: 23 minutes [IQR: 19-30] vs. TM: 24 minutes [IQR: 19-28]), $p=0.70$, with $95 \% \mathrm{CI}(-2.18$ to 3.24$)$ between an OB VN and a TM-based VN on the MSU. 
One of the main considerations in the design of a MSU is the configuration and placement of the CT scanner. Portable CT scanners (CereTom, NeuroLogica-Samsung Corporation, Danvers, MA; Somatom Scope, Siemens, Erlangen, Germany) can be installed across the back wall of the MSU, with special brackets to lock the CT scanner in place during transport. Depending on the type of CT scanner and space allocation, additional enhancements are generally necessary to make the power and charging circuitry more robust, including an upgrade to the standard $\mathrm{OB}$ generator to provide additional power for operating the CT scanner. Eligible patients for tPA are positioned on a gurney which is then raised to align the patient's head with the scanner when performing the CT scan. A standard 8-slice, 5-mm configuration is obtained for each patient and is available for immediate viewing on a portable laptop by the OB VN or readily transmittable to the TM VN and destination hospital via a cloud-based picture archiving and communication system. ${ }^{27}$ Direct comparison of CereTom images with those from a standard clinical CT scan have shown a high comparability of CereTom images in terms of diagnostic accuracy and reliability versus a standard clinical CT scanner. ${ }^{33}$ To safeguard radiation exposure and ensure certainty of obtaining quality CT images, the MSU requires staffing a CT technologist. As a cost-saving measure, CT technologists can be cross-trained as emergency medical technicians to assist the MSU paramedic/nurse when an OB $\mathrm{VN}$ is not available and/or can become also highly skilled in other forms of imaging modalities. Essential point-of-care testing, including fingerstick glucose, international normalized ratio, and creatinine, is readily obtainable and accessible on the MSU, without causing any treatment delays.
Establishing MSUs can be a challenging venture and requires prudent financial investment. The ideal structure and operational design of the MSU will depend on regional/ local terrains, bureaucratic regulations, as well as available economic resources. ${ }^{27}$ Monetary costs to build and fully equip a functional MSU are estimated to be between $\$ 600,000$ and $\$ 2$ million. Although currently available costeffectiveness studies suggest that the expenditure pays off in the long run, ${ }^{34,35}$ the predictive utility is limited. It is anticipated that future research will provide more robust cost-effectiveness analyses. ${ }^{28,31}$

\section{Applications beyond Tissue Plasminogen Activator}

Although reducing time to thrombolysis is a key goal of MSUs, the potential benefits of earlier assessment and MSU intervention are far more expansive. Given recent evidence from clinical trials showing the efficacy of endovascular therapies (ETs) for large vessel strokes, ${ }^{36-38}$ it is vital for triage processes in stroke care to be modified in a manner that promotes efficient triage of ET-eligible patients directly to stroke centers with ET capability. Considering the reduced probability of intra-arterial treatment for every minute of transfer delay to an ET capable hospital, ${ }^{39}$ the identification of patients with large vessel occlusions and/or salvageable penumbra using CT angiography and perfusion on the MSU can save time in the field by bypassing initial transport to non-ET facilities, expedite laboratory testing by using pointof-care testing, ${ }^{40}$ facilitate faster administration of IV tPA, and shorten times to intra-arterial therapy. ${ }^{41}$ The benefits of

\section{Mobile Stroke Unit Process}

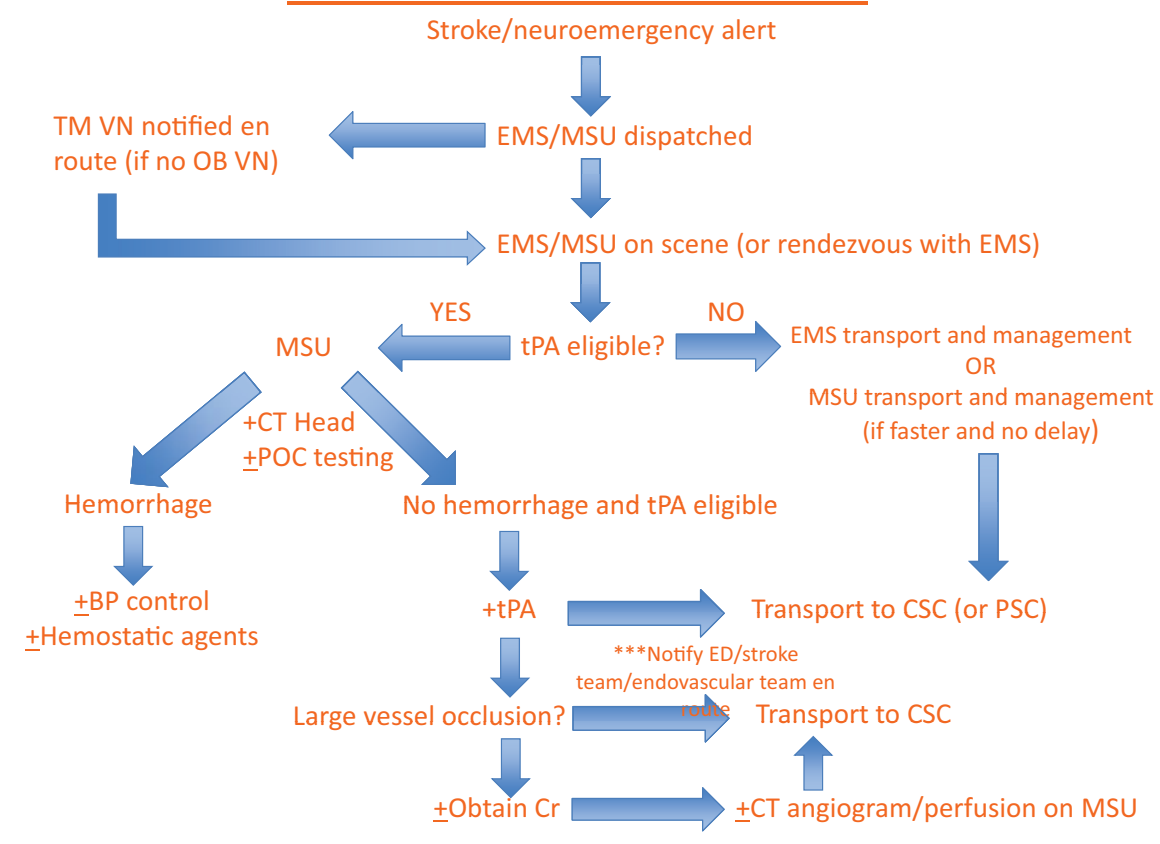

Fig. 1 Algorithm of MSU work flow and triage. Cr, creatinine; CSC, comprehensive stroke center; $\mathrm{CT}$, computed tomography; EMS, emergency medical services; MSU, Mobile Stroke Unit; OB VN, on-board vascular neurologist; POC, point of care; PSC, primary stroke center; TM VN, telemedicine vascular neurologist; tPA, tissue plasminogen activator. 
MSU triage are not only applicable to patients needing ET but are also applicable to virtually any ischemic stroke patient who can benefit from care in hospitals with dedicated stroke units and patients with intracerebral hemorrhages (ICHs) to centers with neurosurgical expertise. ${ }^{42}$ Beyond stroke, efficiency in prehospital triage may also be helpful in patients with head trauma ${ }^{43}$ and other acute neurological emergencies (i.e., status epilepticus) by having patients being diverted to hospitals with expertise in neurological emergencies and critical care. Streamlined triage processes ( - Fig. 1 ) are thus an integral feature of the MSU paradigm.

MSUs also allow for the rapid management of patients with ICH. Since hemorrhage enlargement occurs more frequently early in the course of $\mathrm{ICH}_{1}{ }^{44,45}$ the MSU provides an avenue to promptly administer intravenous antihypertensive medications to control blood pressure and/or other hemostatic agents (i.e., prothrombin complex concentrate for warfarin-associated hemorrhages) and antidotes for specific agents (i.e., idarucizumab for dabigatran) to treat coagulopathies that are associated with ICHs.

MSUs are also an instrumental tool for research spanning broad domains, including biomarkers, ${ }^{46,47}$ neuroimaging, ${ }^{48}$ and cerebral physiology (via the use of noninvasive cerebral blood flow monitoring devices). ${ }^{49}$ The effects of earlier administration of time-sensitive, adjunctive neuroprotective therapies with tPA, along with other hemostatic agents for ICH (i.e., tranexamic acid, factor VIIa) can also be potentially investigated. Dedicated teams, ideally with proficiency in both clinical care and research, will have an invaluable resource given the unique opportunities offered by MSUs.

\section{Future Directions}

The optimal setting (e.g., rural vs. urban) where MSUs can have maximal impact will need to account for innumerable factors, including local EMS systems and protocols, cooperation between hospitals, environmental landscapes, and population dynamics. In urban settings, the proficiency of MSU operations will likely depend on the success of integrating the MSUs within EMS response algorithms and the interplay between EMS responders and hospital systems with differing capabilities of managing stroke patients (i.e., comprehensive stroke centers vs. primary stroke centers, hospitals with neurosurgery, and/or neurocritical care expertise). Significant reductions in time to decision can be made by utilizing a "rendezvous model" in rural settings, where instead of having to wait for patients to be transported to the closest stroke center (which may be distant and require prolonged travel times), MSUs can travel out to meet the incoming ambulance, thereby leading to reductions in time to treatment. This rendezvous approach can also be applied to urban areas and expand the service areas of these units from their base of operation. ${ }^{28}$ Ultimately, the diagnosis of the patient, stroke severity, patient preference, and possibly other region/country-specific rules and regulations will dictate the feasibility of operations of a particular MSU.

One of the keys to the widespread application and success of MSUs will be the standardization of MSU processes and operations across the world. The Prehospital Stroke Treatment Organization (PRESTO) was thus organized in 2016 as a collaboration of international medical providers to improve patient outcomes and promote research across the various spheres of stroke care in the prehospital setting. By facilitating the expansion of MSU programs worldwide, PRESTO can establish a global and cohesive network of stroke care that can link patients, public/municipal resources, and providers alike. On a worldwide scale, MSUs hold great promise with the potential to improve patient outcomes and provide more cost-effective medical care. ${ }^{50}$

\section{References}

1 Rothwell PM, Coull AJ, Silver LE, et al; Oxford Vascular Study. Population-based study of event-rate, incidence, case fatality, and mortality for all acute vascular events in all arterial territories (Oxford Vascular Study). Lancet 2005;366(9499): 1773-1783

2 Murray CJ, Lopez AD. Measuring the global burden of disease. N Engl J Med 2013;369(05):448-457

3 Jauch EC, Saver JL, Adams HP Jr, et al; American Heart Association Stroke Council; Council on Cardiovascular Nursing; Council on Peripheral Vascular Disease; Council on Clinical Cardiology. Guidelines for the early management of patients with acute ischemic stroke: a guideline for healthcare professionals from the American Heart Association/American Stroke Association. Stroke 2013;44(03):870-947

4 Saver JL, Fonarow GC, Smith EE, et al. Time to treatment with intravenous tissue plasminogen activator and outcome from acute ischemic stroke. JAMA 2013;309(23):2480-2488

5 National Institute of Neurological Disorders and Stroke rt-PA Stroke Study Group. Tissue plasminogen activator for acute ischemic stroke. N Engl J Med 1995;333(24):1581-1587

6 Hacke W, Donnan G, Fieschi C, et al; ATLANTIS Trials Investigators; ECASS Trials Investigators; NINDS rt-PA Study Group Investigators. Association of outcome with early stroke treatment: pooled analysis of ATLANTIS, ECASS, and NINDS rt-PA stroke trials. Lancet 2004;363(9411):768-774

7 Lees KR, Bluhmki E, von Kummer R, et al; ECASS, ATLANTIS, NINDS and EPITHET rt-PA Study Group. Time to treatment with intravenous alteplase and outcome in stroke: an updated pooled analysis of ECASS, ATLANTIS, NINDS, and EPITHET trials. Lancet 2010;375(9727):1695-1703

8 Marler JR, Tilley BC, Lu M, et al. Early stroke treatment associated with better outcome: the NINDS rt-PA stroke study. Neurology 2000;55(11):1649-1655

9 Lansberg MG, Schrooten M, Bluhmki E, Thijs VN, Saver JL. Treatment time-specific number needed to treat estimates for tissue plasminogen activator therapy in acute stroke based on shifts over the entire range of the modified Rankin Scale. Stroke 2009; 40(06):2079-2084

10 Khatri P, Yeatts SD, Mazighi M, et al; IMS III Trialists. Time to angiographic reperfusion and clinical outcome after acute ischaemic stroke: an analysis of data from the Interventional Management of Stroke (IMS III) phase 3 trial. Lancet Neurol 2014;13(06): 567-574

11 Katzan IL, Hammer MD, Hixson ED, Furlan AJ, Abou-Chebl A, Nadzam DM; Cleveland Clinic Health System Stroke Quality Improvement Team. Utilization of intravenous tissue plasminogen activator for acute ischemic stroke. Arch Neurol 2004;61(03): 346-350

12 Lichtman JH, Watanabe E, Allen NB, Jones SB, Dostal J, Goldstein LB. Hospital arrival time and intravenous t-PA use in US Academic Medical Centers, 2001-2004. Stroke 2009;40(12):3845-3850 
13 Agyeman O, Nedeltchev K, Arnold M, et al. Time to admission in acute ischemic stroke and transient ischemic attack. Stroke 2006; 37(04):963-966

14 Evenson KR, Foraker RE, Morris DL, Rosamond WD. A comprehensive review of prehospital and in-hospital delay times in acute stroke care. Int J Stroke 2009;4(03):187-199

15 Fonarow GC, Zhao X, Smith EE, et al. Door-to-needle times for tissue plasminogen activator administration and clinical outcomes in acute ischemic stroke before and after a quality improvement initiative. JAMA 2014;311(16):1632-1640

16 Fonarow GC, Smith EE, Saver JL, et al. Timeliness of tissue-type plasminogen activator therapy in acute ischemic stroke: patient characteristics, hospital factors, and outcomes associated with door-to-needle times within 60 minutes. Circulation 2011;123 (07):750-758

17 Lin CB, Peterson ED, Smith EE, et al. Emergency medical service hospital prenotification is associated with improved evaluation and treatment of acute ischemic stroke. Circ Cardiovasc Qual Outcomes 2012;5(04):514-522

18 Ebinger M, Kunz A, Wendt M, et al. Effects of golden hour thrombolysis: a Prehospital Acute Neurological Treatment and Optimization of Medical Care in Stroke (PHANTOM-S) substudy. JAMA Neurol 2015;72(01):25-30

19 Kim JT, Fonarow GC, Smith EE, et al. Treatment with tissue plasminogen activator in the golden hour and the shape of the 4.5-hour time-benefit curve in the National United States get with the guidelines-stroke population. Circulation 2017;135(02):128-139

20 Van Hooff RJ, Cambron M, Van Dyck R, et al. Prehospital unassisted assessment of stroke severity using telemedicine: a feasibility study. Stroke 2013;44(10):2907-2909

21 Itrat A, Taqui A, Cerejo R, et al; Cleveland Pre-Hospital Acute Stroke Treatment Group. Telemedicine in Prehospital Stroke Evaluation and Thrombolysis: taking stroke treatment to the doorstep. JAMA Neurol 2016;73(02):162-168

22 Fassbender K, Walter S, Liu Y, et al. "Mobile Stroke Unit" for hyperacute stroke treatment. Stroke 2003;34(06):e44

23 Walter S, Kostpopoulos P, Haass A, et al. Bringing the hospital to the patient: first treatment of stroke patients at the emergency site. PLoS One 2010;5(10):e13758

24 Walter S, Kostopoulos P, Haass A, et al. Diagnosis and treatment of patients with stroke in a Mobile Stroke Unit versus in hospital: a randomised controlled trial. Lancet Neurol 2012;11(05):397-404

25 Weber JE, Ebinger M, Rozanski M, et al; STEMO-Consortium. Prehospital thrombolysis in acute stroke: results of the PHANTOM-S pilot study. Neurology 2013;80(02):163-168

26 Kunz A, Ebinger M, Geisler F, et al. Functional outcomes of prehospital thrombolysis in a mobile stroke treatment unit compared with conventional care: an observational registry study. Lancet Neurol 2016;15(10):1035-1043

27 Parker SA, Bowry R, Wu TC, et al. Establishing the first Mobile Stroke Unit in the United States. Stroke 2015;46(05):1384-1391

28 Bowry R, Parker S, Rajan SS, et al. Benefits of Stroke Treatment using a Mobile Stroke Unit compared with standard management: the BEST-MSU study run-in phase. Stroke 2015;46(12):3370-3374

29 Wu TC, Parker SA, Jagolino A, et al. Telemedicine can replace the neurologist on a Mobile Stroke Unit. Stroke 2017;48(02):493-496

30 Gutiérrez JM, Emery RJ, Parker SA, Jackson K, Grotta JC. Radiation monitoring results from the first year of operation of a unique ambulance-based computed tomography unit for the improved diagnosis and treatment of stroke patients. Health Phys 2016;110 (05, Suppl 2):S73-S80

31 Yamal JM, Rajan SS, Parker SA, et al. Benefits of Stroke Treatment delivered using a Mobile Stroke Unit trial. Int J Stroke 2017; 0:1747493017711950
32 Ramadan AR, Denny MC, Vahidy F, et al. Agreement among stroke faculty and fellows in treating ischemic stroke patients with tissue-type plasminogen activator and thrombectomy. Stroke 2017;48(01):222-224

33 Rumboldt Z, Huda W, All JW. Review of portable CT with assessment of a dedicated head CT scanner. AJNR Am J Neuroradiol 2009;30(09):1630-1636

34 Gyrd-Hansen D, Olsen KR, Bollweg K, Kronborg C, Ebinger M, Audebert HJ. Cost-effectiveness estimate of prehospital thrombolysis: results of the PHANTOM-S study. Neurology 2015;84(11): 1090-1097

35 Dietrich M, Walter S, Ragoschke-Schumm A, et al. Is prehospital treatment of acute stroke too expensive? An economic evaluation based on the first trial. Cerebrovasc Dis 2014;38(06): 457-463

36 Berkhemer OA, Fransen PS, Beumer D, et al; MR CLEAN Investigators. A randomized trial of intraarterial treatment for acute ischemic stroke. N Engl J Med 2015;372(01):11-20

37 Goyal M, Demchuk AM, Menon BK, et al; ESCAPE Trial Investigators. Randomized assessment of rapid endovascular treatment of ischemic stroke. N Engl J Med 2015;372(11):1019-1030

38 Goyal M, Menon BK, van Zwam WH, et al; HERMES collaborators. Endovascular thrombectomy after large-vessel ischaemic stroke: a meta-analysis of individual patient data from five randomised trials. Lancet 2016;387(10029):1723-1731

39 Prabhakaran S, Ward E, John S, et al. Transfer delay is a major factor limiting the use of intra-arterial treatment in acute ischemic stroke. Stroke 2011;42(06):1626-1630

40 Walter S, Kostopoulos P, Haass A, et al. Point-of-care laboratory halves door-to-therapy-decision time in acute stroke. Ann Neurol 2011;69(03):581-586

41 Cerejo R, John S, Buletko AB, et al. A Mobile Stroke Treatment Unit for field triage of patients for intraarterial revascularization therapy. J Neuroimaging 2015;25(06):940-945

42 Wendt M, Ebinger M, Kunz A, et al; STEMO Consortium. Improved prehospital triage of patients with stroke in a specialized stroke ambulance: results of the pre-hospital acute neurological therapy and optimization of medical care in stroke study. Stroke 2015;46 (03):740-745

43 Schwindling L, Ragoschke-Schumm A, Kettner M, et al. Prehospital imaging-based triage of head trauma with a Mobile Stroke Unit: first evidence and literature review. J Neuroimaging 2016; 26(05):489-493

44 Fujii Y, Takeuchi S, Sasaki O, Minakawa T, Tanaka R. Multivariate analysis of predictors of hematoma enlargement in spontaneous intracerebral hemorrhage. Stroke 1998;29(06):1160-1166

45 Brott T, Broderick J, Kothari R, et al. Early hemorrhage growth in patients with intracerebral hemorrhage. Stroke 1997;28(01): $1-5$

46 Wendt M, Ebinger M, Kunz A, et al; STEMO Consortium. Copeptin levels in patients with acute ischemic stroke and stroke mimics. Stroke 2015;46(09):2426-2431

47 Rozanski M, Waldschmidt C, Kunz A, et al. Glial fibrillary acidic protein for prehospital diagnosis of intracerebral hemorrhage. Cerebrovasc Dis 2017;43(1-2):76-81

48 Grunwald IQ Ragoschke-Schumm A, Kettner M, et al. First automated stroke imaging evaluation via Electronic Alberta Stroke Program Early CT Score in a Mobile Stroke Unit. Cerebrovasc Dis 2016;42(5-6):332-338

49 Tsalach A, Ratner E, Lokshin S, et al. Cerebral autoregulation realtime monitoring. PLoS One 2016;11(08):e0161907

50 Audebert H, Fassbender K, Hussain MS, et al; PRESTO Group. The PRE-hospital Stroke Treatment Organization. Int J Stroke 2017: 1747493017729268 